\title{
Dopaminergic Microtransplants into the Substantia Nigra of Neonatal Rats with Bilateral 6-OHDA Lesions. II. Transplant- Induced Behavioral Recovery
}

\author{
Guido Nikkhah, ${ }^{1,2}$ Miles G. Cunningham, ${ }^{3}$ Ronald McKay, ${ }^{4}$ and Anders Björklund 1 \\ ${ }^{1}$ Department of Medical Cell Research, University of Lund, S-223 62 Lund, Sweden, ${ }^{2}$ Neurosurgical Clinic, \\ Nordstadt Hospital, D-30167 Hannover, Germany, ${ }^{3}$ Harvard Medical School, Boston, Massachusetts 02115, and \\ ${ }^{4}$ Laboratory of Molecular Biology, NINDS NIH, Bethesda, Maryland 20892
}

Transplants of fetal ventral mesencephalic (VM) dopamine neurons implanted into the substantia nigra in 6-hydroxydopamine (6-OHDA)-lesioned neonatal pups establish axonal connections with the denervated caudate putamen (Nikkhah et al., 1995). In the present study, we have explored the functional capabilities of these animals after they reached adulthood on a battery of spontaneous and drug-induced behavioral tasks. The results demonstrate that unilateral intranigral VM grafts in bilaterally lesioned neonates induce a marked bias in spontaneous- and stress-induced rotation contralateral to the implant not present in the lesion-only controls. Amphetamine and apomorphine induced vigorous contra- and ipsilateral rotation, respectively. Moreover, grafted animals achieved $75 \%$ of the performance level in contralateral skilled forelimb use when compared to normal controls, which was significantly above lesion-only animals (50\% of normal). Spontaneous nocturnal locomotor activity was elevated 2.2-fold in the grafted animals. Sensorimotor orientation and disengage behavior was spared by the neonatal dopamine lesion and unaffected by the grafts.

The level of functional restoration seen in the present study was more extensive than reported previously in neonatally 6-OHDA-lesioned rats where the VM grafts were implanted ectopically into the striatum. However, functional recovery remained incomplete also after intranigral graft placement compared to normal intact animals. The present approach should provide a new promising avenue for the continued exploration of the mechanisms involved in functional recovery and structural repair in the damaged nigrostriatal system.

[Key words: ventral mesencephalic grafts, neonates, functional restoration, sensorimotor behavior, Parkinson's disease, developmental plasticity]

\footnotetext{
Received July 22, 1994; revised Nov. 17, 1994; accepted Nov. 29, 1994.

We gratefully acknowledge the excellent technical support of Gertrude Stridsberg, Ulla Jarl, Alicja Flasch, Agneta Persson, and Sten Nilsson. The authors warmly thank Professor Majid Samii and Professor Robert Schönmayr for their continuous support and Dr. Ivar Mendez for valuable suggestions on the manuscript. This study was supported by grants from Swedish MRC (04X3874), the National Institute of Health (NS-06701), and the Goran Gustafsson Foundation. G.N. was supported by a grant from the Deutsche Forschungsgemeinschaft (DFG Ni 330/1-1).

Correspondence should be addressed to Guido Nikkhah, Neurusurgical Clinic, Nordstadt Hospital, Haltenhoffstrasse 41, D-30167 Hannover 1, Germany. Copyright 1995 Society for Neuroscience $0270-6474 / 95 / 153562-09 \$ 05.00 / 0$
}

Unilateral damage to the adult nigrostriatal dopaminergic system induced by 6-hydroxydopamine (6-OHDA) results in variety of behavioral deficits, including spontaneous and drug-induced rotational asymmetry, unilateral defects in sensorimotor orientation, and disengage behavior as well as impaired forelimb use on the side contralateral to the lesion (for a recent review, see Dunnctt and Robbins, 1992). This syndrome is generally viewed as analogous to the sensorimotor deficits in patients suffering from Parkinson's disease (PD), and is thus commonly used as a rodent model of the disease. If the 6-OHDA lesion is applied bilaterally in adult rats, a severe syndrome develops characterized by aphagia, adipsia, and akinesia (Dunnett et al., 1983b). Most strikingly, a comparable dopamine depletion in neonatal pups does not produce any of these devastating symptoms, although some aspects of spontaneous and drug-induced sensorimotor behavior are impaired (Whishaw et al., 1987; Abrous et al., 1992b).

Grafts of fetal ventral mesencephalic (VM) tissue implanted into the striatum of 6-OHDA-lesioned adult rats are effective in restoring several aspects of this $\mathrm{PD}$-like lesion syndrome, but, as reviewed elsewhere (Herman et al., 1989; Björklund, 1992; Björklund et al., 1994), it is clear that deficits in more complex sensorimotor integrative behaviors remain largely unaffected. It has been suggested that the inability of intrastriatal VM grafts to restore more complex sensorimotor behaviors or the regulatory deficits seen after bilateral 6-OHDA lesions may be due to the lack of appropriate regulatory afferent input to the ectopically placed VM grafts (Dunnett and Robbins, 1992; Herman et al., 1989; Mandel et al., 1990; Montoya et al., 1990). Indeed, certain critical regulatory mechanisms may be accessible to the grafted nigral neurons only if they are placed in their normal location in the substantia nigra (SN) itself. Using a modified microtransplantation approach (Nikkhah et al., 1994c), we have recently found that intranigral VM grafts induce a novel pattern of behavioral effects in the adult rat PD model (Nikkhah et al., 1994b) involving substantial recovery in dopamine agonist-induced turning without any effect on amphetamine-induced turning. This effect was most probably mediated via local dopamine release within the $\mathrm{SN}$, because graft-derived TH-positive fiber outgrowth was confined to the SN. Consistent with previous findings (Björklund et al., 1983b), no rostrally directed axon growth towards the denervated striatum was observed in these rats, despite excellent survival and morphological integration of the grafted TH-positive neurons.

In the preceding companion article (Nikkhah et al., 1995), we 


\begin{tabular}{|c|c|}
\hline Postnatal day & Tests \\
\hline P 1 & $\begin{array}{l}\text { Bilateral intraventricular injection of } \\
6 \text {-OHDA }\end{array}$ \\
\hline P 3 & $\begin{array}{l}\text { Implantation of DA } \\
\text { micrografts into the right } S N\end{array}$ \\
\hline P 21 & $\begin{array}{l}\text { Rotation test } 1 \text { : } \\
\text { Spontaneous rotation } \\
\text { Stress induced rotation } \\
\text { Amphetamine, } 2 \mathrm{mg} / \mathrm{kg} \text { i.p. }\end{array}$ \\
\hline P 28 & Rotation test 2: \\
\hline & $\begin{array}{l}\text { Spontaneous rotation (2nd) } \\
\text { Stress induced rotation (2nd) } \\
\text { Apomorphine, } 0.05 \mathrm{mg} / \mathrm{kg} \text { s.c. }\end{array}$ \\
\hline P 42 & Locomotor activity test \\
\hline P 60 & Skilled forelimb use \\
\hline P 90 & $\begin{array}{l}\text { Sensorimotor orientation } \\
\text { Disengage behavior }\end{array}$ \\
\hline P 95 & $\begin{array}{l}\text { TH and Fos } \\
\text { immunohistochemistry }\end{array}$ \\
\hline
\end{tabular}

Figure 1. Sequence of surgery and behavioral testing. The groups comprise 9 normal, 13 bilateral 6-OHDA-lcsioned, and 13 6-OHDAlesioned and unilaterally VM-grafted animals, which were tested on a range of spontaneous and drug-induced behaviors, as indicated. TH, tyrosine hydroxylase.

report that long-distance axon growth is considerably improved if the fetal VM neurons are implanted into the SN of developing neonatal hosts. In animals subjected to a bilateral 6-OHDA lesion at postnatal day $1(\mathrm{Pl})$ and intranigral VM grafts at $\mathrm{P} 3$, we have obtained evidence of extensive functional reinnervation of parts of the denervated striatal complex on the grafted side (Nikkhah et al., 1995). Here, we report that the neonatal intranigral grafts have distinct effects on behavior, as observed in tests of spontaneous, stress, and drug-induced rotation, locomotor activity, and skilled forelimb use, and that these functional effects are more extensive than those seen either with VM grafts implanted into the SN region in the adult 6-OHDA-lesioned rats (Nikkhah et al., 1994b), or with VM grafts transplanted ectopically into the striatum in neonatal 6-OHDA-lesioned hosts (Snyder-Keller et al., 1989; Herman et al., 1991; Abrous et al., 1993b).

\section{Materials and Methods}

\section{Experimental design}

A total of 51 male and female Sprague-Dawley rats (ALAB, Stockholm, Sweden) were used in the experiments. Litters were reared by the mothers until weaning at $21 \mathrm{~d}$ of age. They were housed under a $12 \mathrm{hr}$ light-dark cycle with free access to food and water, first with their mothers, then in groups of four to six, separated by sex. Animals were allocated into three groups: normal $(n=9)$, 6-OHDA $(n=20)$, and graft $(n=22)$ groups. After the amphetamine rotation test at P21, seven lesioned and nine grafted animals died, most likely due to cardiovascular decompensation. The scheme in Figure 1 represents the time course of the experimental design beginning at postnatal day 1 (P1). The animals were killed at $95 \mathrm{~d}$ of age, and the brains were taken for tyrosine hydroxylase and quantitative Fos immunohistochemistry.
The immunohistochemical results are described in detail in the preceding companion article (Nikkhah et al., 1995).

\section{6-OHDA lesion surgery}

On the day after birth, here denoted $\mathrm{P} 1$, the animals received bilateral intraventricular injections of $2 \times 5 \mu \mathrm{l}$ 6-OHDA $(110 \mu \mathrm{g} / 10 \mu \mathrm{l}$ of 6-OHDA $\mathrm{HCl}$ in $0.2 \mathrm{mg} / \mathrm{ml}$ ascorbic acid/saline; Snyder-Keller et al., 1989) using the coordinates: AP $-0.6, \mathrm{~L} \pm 0.8, \mathrm{~V} 2.1$. Details of the handling and operative procedures are described in detail elsewhere (Cunningham et al., 1993).

\section{Neonatal transplantation surgery}

Microtransplants of dopamine-rich cell suspensions were prepared from ventral mesencephalic (VM) tissue of 14-d-old rat fetuses according to a modified microtransplantation approach (Nikkhah et al., 1994c) based on the cell suspension technique described by Björklund et al. (1983a) and Herman et al. (1986). The tissue was incubated in $0.1 \%$ trypsin/ $0.05 \%$ DNase $/ \mathrm{DMEM}$ at $37^{\circ} \mathrm{C}$ for $20 \mathrm{~min}$, rinsed four times in $0.05 \%$ DNase, and mechanically dissociated using a $1 \mathrm{ml}$ Eppendorf pipette. The tissue was then centrifuged at $600 \mathrm{rpm}$ for $5 \mathrm{~min}$ and the pellet resuspended in $0.05 \%$ DNase/DMEM. The cell number of this suspension was 140,000 cells $/ \mu \mathrm{l}$ and the viability was $>95 \%$, as determined by the trypan blue dye exclusion method. The transplantation surgery was performed at P3, as described in detail elsewhere (Cunningham et al., 1993). Briefly, the pups were fixed in the Cunningham hypothermic miniaturized stereotaxic device (Cunningham and McKay, 1993; Stoelting Co.). The micrografts were implanted using a glass capillary with an o.d. of $50-70 \mu \mathrm{m}$ connected to a $1 \mu$ l Hamilton microsyringe. Two $300 \mathrm{nl}$ deposits of the cell suspension were implanted at (1) A -3.7 (from bregma), $\mathrm{L}+1.6 ; \mathrm{V}-4.3$ (from dura); (2) $\mathrm{A}-4.3, \mathrm{~L}+1.6, \mathrm{~V}$ -4.3 .

\section{Rotation tests}

Spontaneous and stress-induced rotation. At $\mathrm{P} 21$, rotational behavior was monitored in automated "rotometer" bowls according to Ungerstedt and Arbuthnott (1970). Spontaneous turning behavior was recorded for a 14 min period. Afterwards, a paper clip was put on the tail of each animal (about $2 \mathrm{~cm}$ from the tip) for $6 \mathrm{~min}$ while continuously recording their rotational behavior. Both tests were repeated at P28 directly before the apomorphine test.

Amphetamine. At $\mathrm{P} 2 \mathrm{l}$ following the stress-induced rotation test, the animals were given $2 \mathrm{mg} / \mathrm{kg} d$-amphetamine (i.p.) and their rotational behavior was monitored for $30 \mathrm{~min}$.

Apomorphine. One week after the amphetamine rotation test (at P28) animals were given $0.05 \mathrm{mg} / \mathrm{kg}$ apomorphine (s.c.) in the neck, and their rotational behavior was monitored for $30 \mathrm{~min}$.

\section{Locomotor activity test}

Locomotor activity was monitored in photocell activity cages at P42. The individual cages were equipped with $2 \times 3$ parallel photocell beams $1 \mathrm{~cm}$ above the floor level. Interruption of the beams resulted in an incremental count, which was recorded on line by an Archimedes computer. The animals were put into the cages at $1800 \mathrm{hr}$ and tested for 15 hr continuously until $0900 \mathrm{hr}$ the next morning.

\section{Skilled forelimb use}

A modified version of the staircase test (Nikkhah et al., 1993) described by Montoya et al. (1991) was performed on all animals at P60. After $2 \mathrm{~d}$ of food deprivation, the animals were tested over 6 consecutive days. For each test the animals were placed into the test boxes for 15 min. For the first $5 \mathrm{~d}$ the double staircase was baited with $4 \times 1045$ mg chow pellets on each side. On day 6 , the left and right staircase were baited with 40 pellets separately ("forced choice" test), allowing the animals $5 \mathrm{~min}$ for food retrieval on each side. After each test the number of pellets taken and the number of pellets eaten was counted separately.

\section{Sensorimotor orientation and disengage behavior}

Sensorimotor neglect was tested once by a "blind" experimenter, according to the protocol of Mandel et al. (1990). The perioral region on each side of the head was touched repeatedly at $1-2$ sec intervals. The latency of an orienting response was recorded to the nearest second. 
Figure 2. Spontaneous $(A)$, stress-induced $(B)$, amphetamine-induced (2 $\mathrm{mg} / \mathrm{kg}$, i.p.) rotation $(C)$ at $\mathrm{P} 21$, and apomorphine-induced $(0.05 \mathrm{mg} / \mathrm{kg}$, s.c.) rotation at $\mathrm{P} 28(D)$. A significant asymmetry in rotational scores was seen in the grafted group, in the direction contralateral to the intranigral VM transplants, in both the spontaneous, stressinduced and amphetamine-induced turning tests, and in the ipsilateral direction in the apomorphine-induced tcst. (*Significant difference from normal, $p<0.001 ;+$, significant difference from 6-OHDA group, $p<0.001$; one-factor ANOVA with post hoc Fisher PLSD test.) Bars represent group means $\pm \mathrm{SEM}$
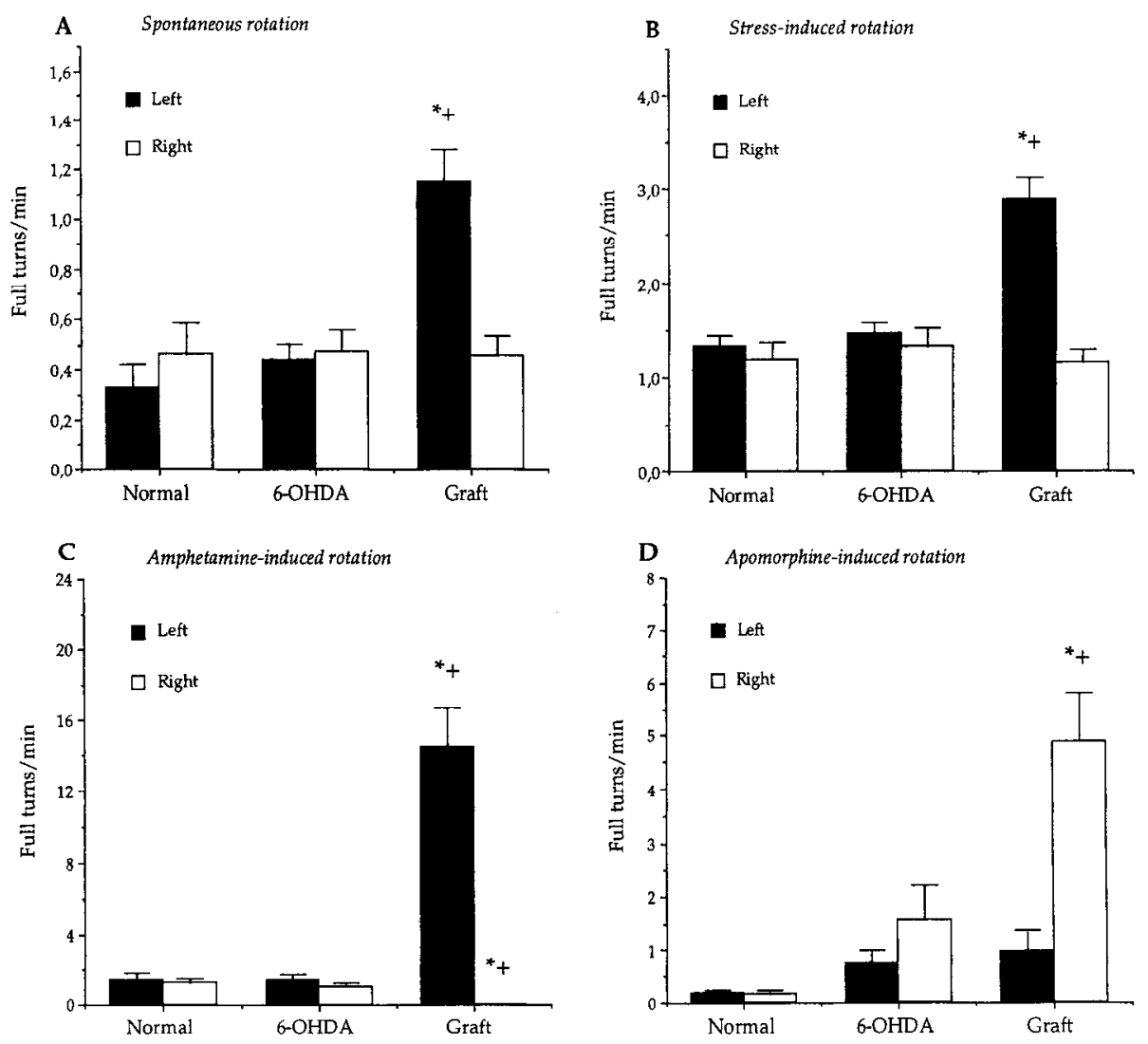

Disengage behavior was assessed as described above, except that the animals were given a piece of milk chocolate to eat while being stimulated periorally. Perioral stimulation was discontinued if the animal did not respond within $180 \mathrm{sec}$. The animals were tested once daily on 2 consecutive days.

\section{Statistical analysis}

Results are expressed as means \pm SEM of the different treatment groups. For statistical evaluation, data was subjected to one-way analysis of variance (ANOVA) and Fisher PLSD post hoc test. Repeated spontaneous and stress-induced rotation tests were statistically analyzed by a two-factor ANOVA. For all statistical tests the significance level was set at $p<0.05$.

\section{Results}

\section{Rotation tests}

Spontaneous rotation. Spontaneous rotation was recorded for a period of $14 \mathrm{~min}$ at 3 weeks postimplantation (P21), as shown in Figure 2A. Normal and 6-OHDA-lesioned rats did not show any motor asymmetry in the rotometer bowls, and they scored 0.3-0.5 full turns/min to either side. Grafted animals (grafted in the right $\mathrm{SN}$ ) demonstrated significantly higher number of turns to the left ( 1.1 turns/min) than to the right $(0.5$ turns $/ \mathrm{min})(p<$ 0.01 ), which was also significantly higher than the left rotation scores for the normal and lesion group $(p<0.001)$. One week after the amphetamine rotation test spontaneous turning was recorded again (data not shown) and found to have increased about twofold from the first test in both the normal and the lesioned animals $(p<0.001)$. Grafted animals showed an increase in left turns by $160 \%$ between the first and the second test, which was significantly higher than in the other two groups $(p<0.001$, two-factor ANOVA).

Stress-induced rotation. Stress-induced rotation was elicited at P21 by putting a paper clip on the tail of each animal and monitored for $6 \mathrm{~min}$. No side difference in rotation response was observed in normal and lesioned animals, both groups scoring 1.3-1.5 turns $/ \mathrm{min} /$ side in response to the stress stimulus, as illustrated in Figure $2 B$. The grafted animals were markedly asymmetric, showing a significantly higher rotation score to the left side, 2.9 turns/min, i.e., in the direction contralateral to the graft, which was significantly higher than in the other two groups $(p<0.001)$. In contrast to the spontaneous rotation, there was no significant increase in stress-induced rotation scores in any of the groups between the first and the second test (data not shown).

Amphetamine-induced rotation. The ipsi- and contralateral rotation response to amphetamine ( $2 \mathrm{mg} / \mathrm{kg}$, i.p.) at $\mathrm{P} 21$ is shown in Figure $2 C$. Animals from the normal and lesion group scored on average $1.0-1.5$ turns $/ \mathrm{min}$ over the $30 \mathrm{~min}$ test period to either side. Grafted animals turned almost exclusively to the side contralateral to the implants, with a mean of 14.5 turns $/ \mathrm{min}$. Ipsilateral turning scores in this group was 0.1 turns $/ \mathrm{min}$, which was significantly below the rate seen in the other two groups ( $p$ $<0.001)$.

Apomorphine-induced rotation. There was an overall trend towards an increased turning response to apomorphine $(0.05 \mathrm{mg} /$ $\mathrm{kg}$, s.c.) in the lesioned animals compared to the normal group, as demonstrated in Figure $2 D$, but this increase did not reach significance. Rotation scores were 0.2 turns/min (over $30 \mathrm{~min}$ ) and 0.7-1.6 turns/min in either direction for the normal and lesion group, respectively. There was a significant increase in rotation asymmetry in the grafted animals, from $1 \mathrm{turn} / \mathrm{min}$ contralateral to the implant to 4.9 turns/min ipsilaterally $(p<$ 0.001 ). This increase in the rate of ipsilateral turns in the grafted animals was also significant when compared to the normal and lesion group $(p<0.001)$. 


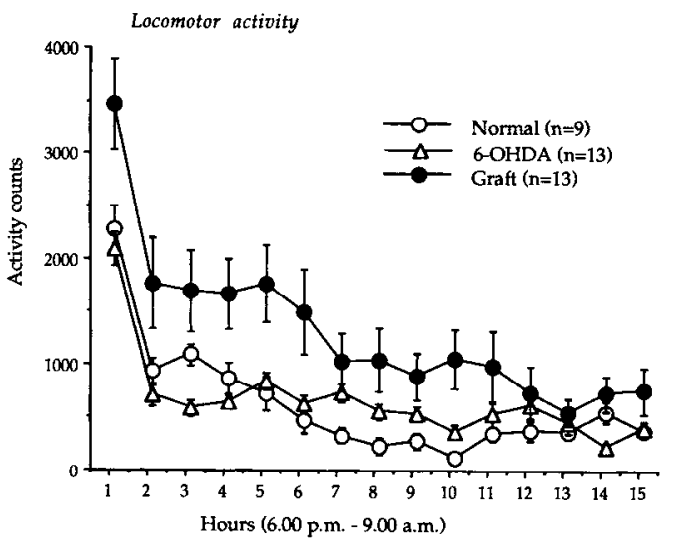

Figure 3. Time course of locomotor activity at $42 \mathrm{~d}$ of age. Grafted animals were hyperactive throughout the whole night when compared to the normal and 6-OHD $\Lambda$ lesion group ( $p<0.01$, one-factor $\Lambda$ NOVA with post hoc Fisher PLSD test). Each point represents the mean score (mean \pm SEM) over $1 \mathrm{hr}$.

\section{Iocomotor activity}

Spontaneous nocturnal activity was monitored for $15 \mathrm{hr}$ in all three groups at P42, as shown in Figure 3. Animals from all groups were most active during the first hour (which coincided with the onset of the dark period at $1800 \mathrm{hr}$ ), demonstrating a strong exploratory behavior. Thereafter, locomotor activity counts decreased to a plateau level in the normal and lesion groups. The grafted animals showed a more gradual decrease in locomotor activity, not reaching their baseline activity until $7 \mathrm{hr}$ of testing. They exhibited an initial burst of exploratory behavior $62 \%$ above the activity counts measured in the normal and lesion groups $(p<0.01)$. The grafted animals remained significantly hyperactive throughout the whole test period, although at later time bins (at 12-15 hr when the light had been switched on) the difference compared to the lesion and normal groups was less pronounced. During the entire test period the grafted animals were, on average, 2.2 times more active than lesion and normal animals $(p<0.01)$. No significant difference in spontaneous nocturnal locomotor activity was observed between the lesion and the normal group, although there was a trend towards hyperactivity in the lesion group for most parts of the night compared to the normal group.

\section{Skilled forelimb use}

During the first 5 test days, where the double staircase was baited with 40 food pellets on each side, normal animals (at P60) quickly acquired the reaching task and successfully retrieved more than $75 \%$ of all pellets with either forelimb from day 3 onwards (Fig. 4A,B). In the bilaterally 6-OHDA-lesioned as well as in the unilaterally grafted animals the number of successfully collected pellets ("pellets eaten") was significantly reduced over the whole $5 \mathrm{~d}$ test period $(p<0.01$ ). At day 5 the lesion-only animals achieved $54 \%$ and $66 \%$ for the left and right paw, respectively, compared to the scores of the normal group. By contrast, neither the 6-OHDA-lesioned rats nor the grafted animals were impaired on the number of attempts made (see "pellets taken" in Fig. 4C). The grafted animals successfully retrieved $64 \%$ (left) and $57 \%$ (right) of the number of pellets collected by the normal animals. Performance levels in the lesion-only and the grafted animals did not differ statistically during the first 5 $\mathrm{d}$ of testing, although it is noteworthy that in the grafted group the learning curve for the left forelimb, i.e., the limb contralateral to the grafts, tended to be above that of the lesion-only group and below that of the lesion-only rats for the right paw.

The results for day 6 , when each side of the staircase was baited separately, are illustrated in Figure $4, C$ and $D$. There was no significant difference in the number of pellets taken (i.e., pellets that had been touched and removed from the four levels of the staircase) among the three groups for either forelimb (Fig. $4 C$ ). However, a substantial deficit was seen in the number of pellets eaten (i.e., pellets that had been successfully retrieved) (Fig. 4D). The 6-OHDA-lesioned group successfully retrieved

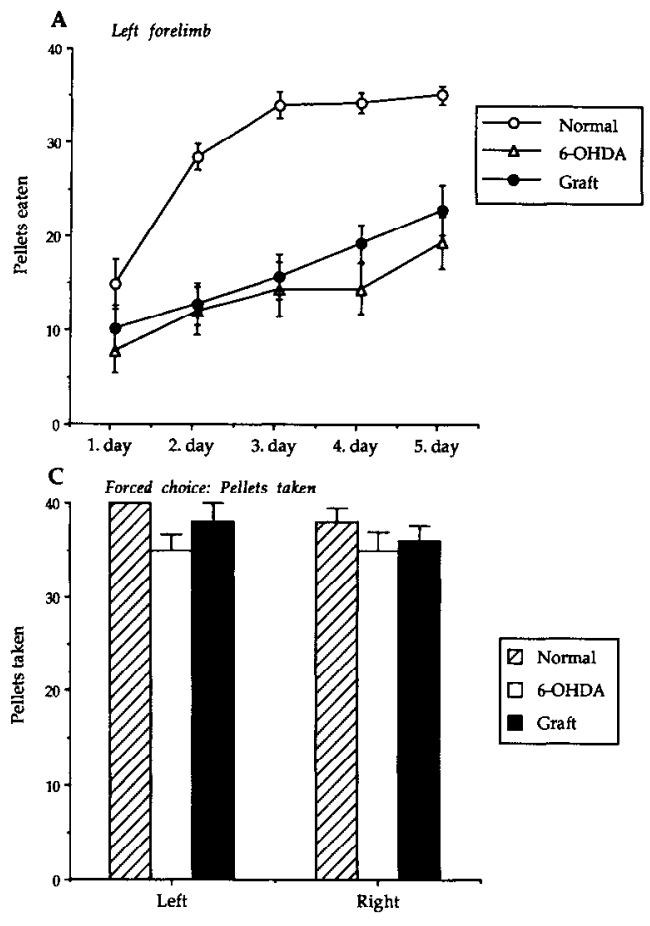

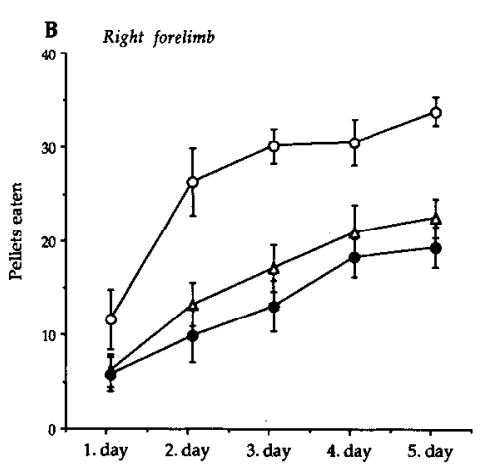

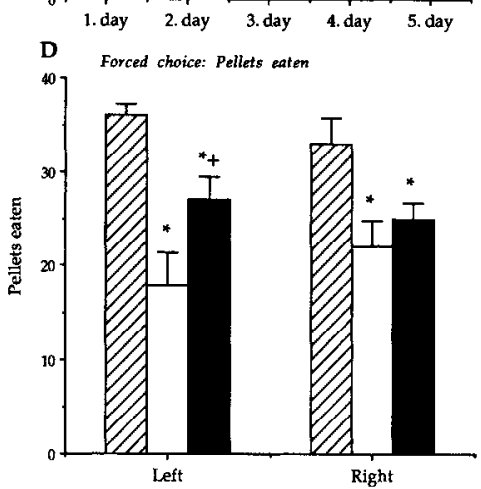

Figure 4. Mean number of pellets eaten with the left $(A)$ and right $(B)$ forelimb during 15 min over the first 5 $\mathrm{d}$ of the paw-reaching test, when both staircases were baited with 40 pellets on each side. There is a clear impairment on both sides in the lesioned and grafted animals compared to the normal group. Mean number of pellets taken $(C)$ and eaten $(D)$ in the forced choice test on day 6 , when each side of the staircase was baited separately with 40 pellets, and the test was run for 5 min on each side only. There was a partial, but significant improvement of the graft group over the 6-OHDA-lesioned rats in the number of pellets eaten with the left, but not with the right forelimb. (*Significant difference from normal, $p<0.001$, except for lesion vs. normal right, $p<0.05 ;+$, significant difference from 6-OHDA group, $p$ $<0.001$; one-factor ANOVA with post hoc Fisher PLSD test.) Bars represent group means \pm SEM of 9 normals, 13 6-OHDA-lesioned, and 13 6-OHDAlesioned and grafted rats. 
only $18(50 \%$ of normal, $p<0.001)$ and $22(67 \%$ of normal, $p$ $<0.05$ ) pellets with the left and right forelimb, respectively. The success rate in the grafted animals was $75 \%$ of normal on the left side, which was significantly better than in the lesiononly animals $(p<0.001)$. The grafted rats, however, remained significantly impaired compared to the normal controls on both sides $(p<0.001)$.

\section{Sensorimotor behavior}

In contrast to the syndrome seen in rats lesioned with 6-OHDA during adulthood (Mandel et al., 1990; Nikkhah et al., 1993), there was no significant increase in response latency in the neonatally 6-OHDA-lesioned animals, either in the simple sensorimotor orientation test or in the disengage behavior test, when the rats were tested identically while eating (data not shown). Response latencies after perioral stimulation were similar, in the range of $12 \mathrm{sec}$, in both tests in all three groups with no sig nificant difference among them.

\section{Discussion}

The present results provide evidence that fetal VM grafts implanted homotopically into the substantia nigra (SN) in 6-OHDA-lesioned neonatal pups can exert significant functional effects on dopamine-dependent behaviors that are more extensive than those seen either after ectopic intrastriatal VM grafts in neonatal 6-OHDA-lesioned hosts, or after VM grafts implanted into the SN region of adult 6-OHDA-lesioned rats.

Two small deposits of $0.3 \mu \mathrm{l}$ (each containing about 40,000 cells) were implanted unilaterally into the bilaterally 6-OHDAlesioned $\mathrm{SN}$ at $\mathrm{P} 3$. As described in the preceding companion article (Nikkhah et al., 1995), the grafted dopaminergic neurons survived and integrated well into the 6-OHDA-lesioned SN. Graft-derived TH-positive fibers demonstrated long-distance growth along the nigrostriatal pathway and established a new TH-positive terminal network in parts of the ipsilateral caudate putamen, which was paralleled by a normalization of dopamine agonist-induced Fos expression in the striatal target neurons.

Graft-dependent functional effects were observed in several behavioral tests, including spontaneous, stress-, amphetamine-, and apomorphine-induccd rotation and locomotor activity, and a partial effect was obtained also on skilled forelimb use on the side contralateral to the transplant.

In previous studies on ectopically placed intrastriatal VM grafts in 6-OHDA-lesioned neonatal rats the behavioral effects of VM transplants have been limited to drug-induced motor asymmetry and locomotor activity (Snyder-Keller et al., 1989; Abrous et al., 1990, 1993b,c; Herman et al., 1991). In addition, bilateral intrastriatal VM grafts implanted neonatally have been shown to protect against the aphagia, adipsia, and akinesia induced by a subsequent adult bilateral 6-OHDA lesion (Schwarz and Freed, 1987; Rogers and Dunnett, 1989, 1990; Rogers et al., 1990).

\section{Behavioral deficits induced by the neonatal 6-OHDA lesion}

Bilateral intraventricular injection of 6-OHDA at P1 induced a substantial impairment in skilled forelimb use, as tested when the animals had reached adulthood. The bilateral lesion did not induce any rotational bias, nor did it affect locomotor activity when compared to normal intact controls. No ingestive dysfunctions or sensorimotor deficits could be observed despite the fact that the caudate putamen complex was extensively denervated of its $\mathrm{TH}$-positive afferents. As described in the preceding companion article (Nikkhah et al., 1995) TH-positive neurons remained in the VTA, and the TH-positive projections to several mesolimbic regions (nucleus accumbens, olfactory tubercle, amygdala) were partially spared. This is in accordance with previous studies (Bruno et al., 1984; Abrous et al., 1990; Luthman et al., 1994), which have shown that neonatal 6-OHDA lesions fail to induce some of the severe impairments seen after bilateral 6-OHDA lesions in adult rats (Dunnelt et al., 1983b; Dunnett and Robbins, 1992). Nevertheless, neonatal 6-OHDA lesions do produce significant behavioral deficits in response to homeostatic challenges (Bruno et al., 1986), spatial discrimination (Feeser and Raskin, 1987), skilled forelimb and tongue use, and limb posture (Whishaw et al., 1987).

\section{Rotation}

The graft-induced effects on amphetamine- and apomorphineinduced turning seen here are similar to what has previously been observed in animals receiving unilateral VM grafts into the striatum as neonates (Snyder-Keller et al., 1989; Herman et al., 1991; Abrous et al., 1993b,c). Animals with unilateral intranigral VM grafts exhibited, in addition, a clear spontaneous and stressinduced rotational bias towards the side contralateral to the implant prior to any drug challenge, unlike normal and lesion-only rats, which showed no side bias. Such effects have not been observed previously in neonatal 6-OHDA-lesioned rats with ectopic intrastriatal VM grafts (Carder et al., 1988; Snyder-Keller et al., 1989). Stress-induced turning after intrastriatal VM grafts in neonates has been described in a number of studies (Carder et al., 1987, 1988; Snyder-Keller et al., 1989), but it has been shown that the stress-induced effect seen in these animals only developed after prior exposure to amphetamine in the same environment (Snyder-Keller and Lund, 1990). Recently, Annett et al. (1993) have demonstrated, that stress-induced rotation in rats lesioned and grafted as adults is due to a "conditioned response between amphetamine and the environment" rather than to a priming effect of VM grafts by amphetamine. Such conditioning was clearly not necessary for the expression of stress-induced turning in the present rats with neonatal intranigral VM grafts.

It is known that DA agonist-induced turning can be induced via receptors located in both striatum and SN (Cheramy et al., 1981; Robertson et al., 1992). It is likely therefore that the effects of the neonatal intranigral VM grafts on rotation could be mediated through release of dopamine, not only from the terminals innervating the striatum, but also from the cells and dendrites within the SN itself. In fact, we have found that dopamine agonist-induced rotational asymmetry in adult unilateral 6-OHDA-lesioned rats can be ameliorated by VM grafts intplanted into the $\mathrm{SN}$, whereas amphetamine-induced turning was not affected (Nikkhah et al., 1994b). This is in contrast to the results obtained in the present study, where amphetamine induced a vigorous contralateral turning response in the neonatally grafted animals. This has also been reported for intrastriatal VM grafts both in 6-OHDA-treated neonates (Carder et al., 1987, 1988; Snyder-Keller et al., 1989; Herman et al., 1991; Abrous et al., 1993b,c) as well as in bilaterally 6-OHDA-lesioned adult rats (Björklund et al., 1981; Dunnett et al., 1983b). This indicates that graft-derived dopamine release within the striatum, and not in the $\mathrm{SN}$, is responsible for the induction of turning in response to amphetamine. The intensity of amphetamine-induced turning seen here (about 15 turns/min) is similar to that observed in rats with complete $(>97 \%$ ) unilateral 6-OHDA lesions of the nigrostriatal pathway, whereas the amphetamine- 
induced striatal Fos expression (see preceding article, Nikkhah et al., 1995) was significantly higher than normal. This exaggerated Fos response may, at least in part, be due to a 6-OHDA lesion-induced hyperactivity of the glutamatergic corticostriatal afferents, as proposed by Cenci and Björklund (1994).

Apomorphine induced ipsilateral turning in the intranigral VM-grafted neonatal animals. Similar effects are seen with unilateral VM grafts in the striatum, both in neonates and adults with bilateral 6-OHDA lesions (Dunnett et al., 1983b; Herman et al., 1991), suggesting that the effect is mediated by a normalization of DA receptor sensitivity in the striatum ipsilateral to the SN grafts. This is supported by the downregulation of the apomorphine-induced Fos response on this side (Nikkhah et al., 1995). It should be pointed out, however, that apomorphine-induced turning can be markedly influenced also by DA release within the nigra itself (Nikkhah et al., 1995).

\section{Locomotor activity}

Both the caudate putamen and the nucleus accumbens have been suggested to play a fundamental role in the regulation of locomotor activity. Dopamine levels in the caudate putamen are more closely linked to the posture and direction of movements, while accumbens dopamine levels seemed to be related to the overall locomotor activity (Dunnett and Robbins, 1992). The bilateral intraventricular 6-OHDA lesion in the neonates did not result in a significant change in locomotor activity, as assessed at 6 weeks of age in the present study, although there was a trend towards hyperactivity in the lesioned animals corroborating the results of earlier studies (Rogers and Dunnett, 1989; Abrous et al., 1993b). The intranigral VM grafts induced a twofold increase in spontaneous nocturnal locomotor activity. Similar effects have been seen with bilateral VM grafts into the striatum in neonates (Abrous et al., 1993b), as well as with bilateral VM grafts into the frontal cortex and nucleus accumbens in adult recipients (Cenci et al., 1994). In the latter microdialysis study, Cenci et al. (1994) found that the increased spontaneous locomotor activity was significantly correlated with graft-derived dopamine release in the reinnervated frontal cortex (which was higher than normal). Although it has not been possible to assess to what extent the intranigral VM grafts project to cortical and limbic areas in the present animals, it is conceivable that the hyperlocomotor response seen here may be due to excessive dopamine release in critical forebrain areas reinnervated by the grafted neurons.

\section{Sensorimotor orientation}

The sensorimotor orientation response was unaffected by the neonatal 6-OHDA lesion, which is in agreement with previous findings (Whisaw et al., 1987). Interestingly, not only baseline sensorimotor orientation but also "disengage" behavior, i.e., the ability of the rat to orient towards external sensory stimuli while eating, was unaffected. This is in marked contrast to the spontaneous recovery seen in adult rats with subtotal lesions of the mesotelencephalic dopamine system where baseline sensorimotor orienting responses may recover completely while disengage behavior remains impaired (Schallert and Hall, 1988). This discrepancy suggest that functional sparing of sensorimotor orienting behavior after either neonatal or adult 6-OHDA lesions may depend on different compensatory mechanisms operating during development and in adulthood.

\section{Skilled forelimb use}

Deficits in skilled forelimb use in the neonatally 6-OHDA-lesioned rats amounted to about 50\% of the performance level of normal controls, as assessed by a modified version of the staircase test of Montoya et al. (1991). The lesion-induced deficits seen in the neonatally lesioned rats, when tested as adults, was comparable in magnitude to the impairment observed in our previous study in animals subjected to a unilateral 6-OHDA lesion in adulthood (Nikkhah et al., 1993). These results are corroborated by a study of Whishaw et al. (1987) showing a similar decrease in the number of successful skilled forelimb reaches after bilateral intraventricular 6-OHDA lesion in neonates. A selective unilateral 6-OHDA injection into the medial forebrain bundle in neonatal pups, however, seems to produce less pronounced reaching deficits compared to the same lesion in adulthood (Abrous et al., 1992). It is interesting to note that paw reaching was markedly impaired in the neonatally lesioned rats despite the fact that sensorimotor behavior (tested in the same rats) was unaffected. This indicates that the performance deficit in the paw-reaching test is more complex and reflects disturbances that go beyond that of a sensorimotor initiation impairment. This is consistent with the observation that the 6-OHDAlesioned rats were not impaired on the number of attempts made by either paw in the staircase test.

The neonatal intranigral VM grafts significantly improved the level of performance in the contralateral paw, although the effect was only seen in the final stage of the test, when only one staircase was baited, and remained partial. This is interesting in light of previous studies, which have failed to demonstrate any beneficial effects of intrastriatal grafts on skilled paw use, either in neonates (Abrous et al., 1993c) or in adults (Montoya et al., 1990; Abrous et al., 1993a). On basis of these observations it has been suggested that ectopic intrastriatal VM grafts might be insufficient to restore skilled motor behavior in the absence of physiologically relevant regulatory afferents (Montoya et al., 1990; Abrous et al., 1993a,c). The recovery of skilled forelimb use obtained by intranigral VM grafts in the present study may, at least partly, support such a hypothesis. It should be noted, however, that intrastriatal VM grafts may be able to improve skilled forelimb use to a similar degree in unilaterally lesioned adults rats provided that an extensive dopaminergic reinnervation throughout the head of the caudate putamen is achieved. This has been possible to obtain by using intrastriatal VM microtransplants distributed as multiple deposits over a large area of the striatum which is likely to improve graft-host integration (Nikkhah et al., 1993). Similarly, restoration of successful paw use has also been reported in 6-OHDA-lesioned marmosets following multiple intrastriatal VM grafts (Annett et al., 1994).

\section{Functional limitations of intranigral VM grafts}

The extent of graft-induced functional recovery seen in the present study may, in part, be due to the homotopic placement of the transplant and, in part also to the ability of the intranigral VM transplants to establish axonal connections with the denervated caudate putamen. The functional recovery seen with homotopic neonatal intranigral VM grafts appcars to be more extensive than after ectopic grafts implanted into the striatum (see Fig. 5). However, functional restoration was clearly incomplete and the spontaneous locomotor behavior and cellular changes in response to amphetamine challenge were abnormal (Nikkhah et al., 1995). There may be several reasons why recovery of function is limited with the present approach. 
Figure 5. Schematic representation of three alternative transplantation approaches to achieve functional restoration of the lesioned (here absent) nigrostriatal dopamine pathway. To demonstrate the main point, the nigrostriatal connectivity has been greatly simplified. The grafted dopaminergic neurons are highlighted in the black box. A represents the principal anatomical connections of ectopic intrastriatal VM grafts in adult and neonatal hosts. $B$ illustrates the likely anatomical connections of intranigral VM grafts in adult hosts. $C$ illustrates the proposed pathway reconstruction obtained by intranigral VM grafts in neonatal recipients, as studied in the present report. *Indicate possible synaptic connections, which have not been demonstrated so far. $C t x$, cortex; $C P U$, caudate putamen; $S N p c$, substantia nigra pars compacta; $S N p r$, substantia nigra pars reticulata; $S T N$, subthalamic nucleus.

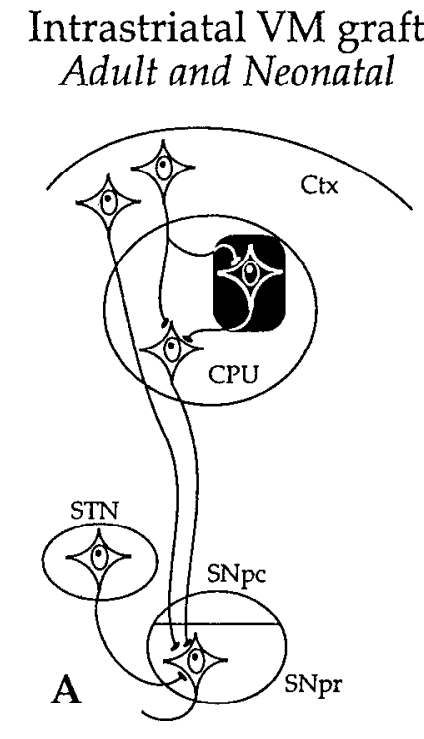

$\underset{\text { Neonatal }}{\text { Intranigral VM graft }}$
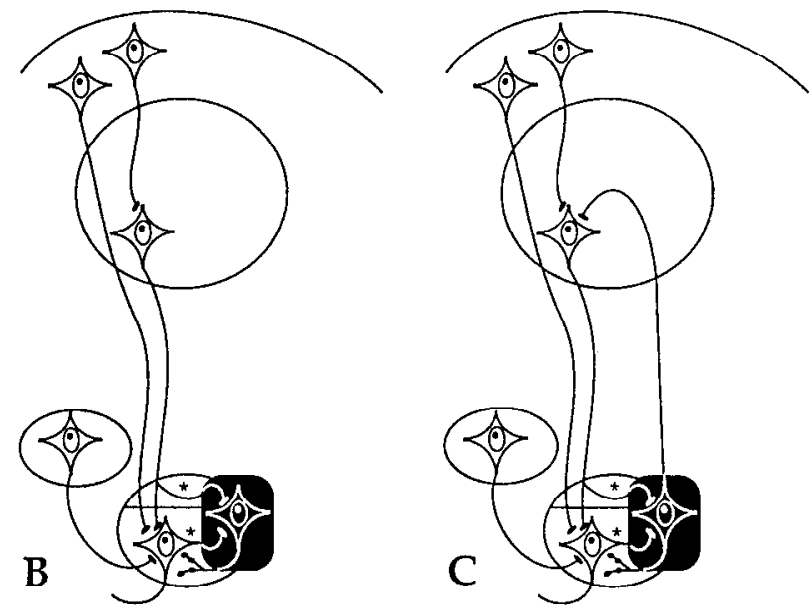

A first explanation comes from the time delay between lesion (P1), implantation (at P3) and the subsequent maturation of the grafted VM neurons, which is likely to result in a delay of at least 1 week between the loss of the intrinsic nigrostriatal projection and the establishment of a new innervation of the host striatum. It has been shown that a delayed striatal reinnervation by intrastriatal dopaminergic grafts cannot prevent the onset of compensatory mechanisms, such as the lesion-induced serotonergic striatal hyperinnervation (Snyder-Keller et al., 1989), which, in this case, may not be responsible for the functional sparing observed in these animals (Bruno et al., 1987). It seems possible that intrinsic compensatory mechanisms, active in the neonatal brain, might, on one hand, result in sparing of some behaviors (e.g., sensorimotor orientation and disengage behavior), but at the same time evoke a state of the lesioned brain that is more refractory to the influence of the VM grafts.

A second explanation comes from the developmental mismatch between the VM grafts, taken from E14 fetuses, and the developmental state of the neonatal host brain, which would explain the inability of the VM transplants to fully reconstruct the SN. Although TH-positive fibers from the grafted neurons were able to establish axonal connections with the striatum, the grafted dopaminergic neurons did not migrate to a significant extent to reform the characteristic pars compacta structure of the lesioned host $\mathrm{SN} p \mathrm{c}$. As described in the preceding companion article (Nikkhah et al., 1995), the grafted TH-positive neurons were well integrated with the host tissue but most of the cells occurred outside the $\mathrm{SNpc}$, most notably in the pars reticulata, the cerebral peduncle, and the subthalamic region. This highlights the possibility that outside a limited developmental time window (which is likely to be during the third week of the fetal period) the developmental cues that guide the developing nigral neurons to their normal location are no longer available to the implanted fetal VM neurons.

Finally, the VM grafts studied here were limited to one hemisphere, leaving the contralateral $\mathrm{SN}$ and striatum denervated. This imbalance may have exerted certain constraints over the level of functional restoration also in the grafted hemisphere. Further studies using bilateral intranigral transplants in the neonatal model are thus warranted.
Advantages of homotopic graft placement in the rat PD model The present study has extended previous approaches towards a more complete reconstruction of the lesioned nigrostriatal circuitry (Fig. 5). Ectopic intrastriatal VM grafts in adult hosts have been shown to establish a synaptic afferent (Bolam et al., 1987; Doucet et al., 1989; Fisher et al., 1991) and efferent connectivity (Freund et al., 1985; Mahalik et al., 1985; Mendez et al., 1991) with cortical and striatal neurons (Fig. 5A). Their electrophysiological maturation and responsiveness, however, remains, in certain respects, immature and incomplete (Fisher et al., 1991). The functional constraints secn with intrastriatal graft placement, most apparent in the failure to restore more complex sensorimotor behaviors, have largely been attributed to the lack of sufficient restoration of dopamine levels and/or the ectopic implantation site (Björklund, 1992; Björklund et al., 1987, 1994; Herman et al., 1989). The microtransplantation approach has made it possible to achieve increased dopamine-tissue levels and more extensive TH-positive fiber oulgrowth from multiple VM deposits spread throughout the head of the caudate putamen (Nikkhah et al., 1994a). However, graft-derived TH-positive reinnervation was confined to the telencephalon, leaving the SN completely devoid of a dopaminergic innervation. These microtransplanted rats showed partial recovery in skilled forelimb use and disengage behavior (Nikkhah et al., 1993), but they were still significantly impaired compared to normal intact control animals. Intrastriatal VM grafts in neonatal rats do not seem to provide any additional functional recovery despite signs of better morphological graft-host integration at the ectopic implantation site (Snyder-Keller et al., 1989; Herman et al., 1991; Abrous et al., 1993b,c).

Intranigral VM grafts, which most probably act via release of dopamine from dendritic processes within the $\mathrm{SN}$ itself (Fig. $5 B$ ), have recently been shown to be capable of reducing dopamine-agonist induced rotation in unilaterally $6 \mathrm{OHD} \Lambda$ - $\mathrm{e}$ sioned adult rats to a degree comparable to that seen with intrastriatal VM grafts (Nikkhah et al., 1994b). In this case, however, no reinnervation of the denervated striatum or other major target areas occurred, which might explain the absence of any effect on the amphetamine-induced rotation by the intranigral VM grafts. 
The anatomical connection of the homotopically placed intranigral VM grafts with the denervated striatum in neonatal hosts (Fig. 5C) provide a new model for the study of transplantinduced behavioral recovery. The neonatal intranigral VM grafts exert marked functional effects on spontaneous and stress-induced rotation, and they have, in addition, a capacity to induce partial recovery in skilled forelimb use. Since these effects appear to go beyond what has previously been possible to obtain with ectopic intrastriatal VM grafts in the neonatal model, it is clearly warranted to explore further the importance of the restored nigrostriatal connectivity for the observed functional effects.

In conclusion, the results show that the intranigral fetal VM grafts exert distinct and marked effects on both drug-induced and spontaneous motor behavior in the neonatal 6-OHDA-lesioned recipients, when tested as adults. In combination with the immunohistochemical observations in the preceding companion article (Nikkhah et al., 1995), these data strongly indicate that the nigrostriatal projection established by the grafted TH-positive neurons is, indeed, functional and capable of modifying the rats' ongoing motor behavior via dopamine release from the graft-derived axonal terminals in the reinnervated striatum, and possibly also in other forebrain targets that may receive projections from the intranigral VM grafts. These observations are consistent with findings in other neonatal transplantation models, such as the retinotectal system (Lund et al., 1990, 1992) and the spinal cord (Iwashita et al., 1994), where partial reformation of functional pathways by fetal neural transplants have been possible to achieve. Indeed, the ability of intranigral VM grafts to form axonal connections of with the denervated host striatum, as presented here, might enable grafted dopaminergic neurons to establish some of the critical control mechanisms by which they normally influence the processing of basal ganglia information (Gerfen, 1992; Robertson, 1992). If so, this approach may have important implications for the further development of the clinical neurotransplantation strategies currently pursued (Lindvall et al., 1990; Freed et al., 1992; Widner, 1992) in order to restore functional abilities in patients with Parkinson's disease.

\section{References}

Abrous N, Choulli K, Simon H, LeMoal M, Herman JP (1990) Behavioural effects of intracerebral dopaminergic grafts after neonatal destruction of the mesencephalic dopaminergic system. Prog Brain Res 82:481-487.

Abrous DN, Wareham AT, Torres EM, Dunnett SB (1992) Unilateral dopamine lesions in neonatal, weanling and adult rats: comparison of rotation and reaching deficits. Behav Brain Res 51:67-75.

Abrous DN, Shaltot A, Torres EM, Dunnett SB (1993a) Dopaminerich grafts in the neostriatum and/or nucleus accumbens: effects on drug-induced behaviours and skilled paw-reaching. Neuroscience 53: $187-197$.

Abrous DN, Choulli K, Rouge-Pont F, Simon H, Le Moal M, Herman JP (1993b) Effects of intracerebral dopaminergic grafts on behavioural deficits induced by neonatal 6-hydroxydopamine lesions of the mesotelencephalic dopaminergic pathway. Neuroscience 54:499-511.

Abrous DN, Torres EM, Dunnett SB (1993c) Dopaminergic grafts implanted into the neonatal or adult striatum: comparative effects on rotation and paw reaching deficits induced by subsequent unilateral nigrostriatal lesions in adulthood. Neuroscience 54:657-668.

Annett LE, Reading PJ, Tharumaratnam D, Abrous DN, Torres EM, Dunnett SB (1993) Conditioning versus priming of dopaminergic grafts by amphetamine. Exp Brain Res 93:46-54.

Annett LE, Martel FL, Rogers DC, Ridley RM, Baker HF, Dunnett SB (1994) Behavioral assessment of the effects of embryonic nigral grafts in marmosets with unilateral 6-OHDA lesions of the nigrostriatal pathway. Exp Neurol 125:228-246.

Björklund A (1992) Dopaminergic transplants in experimental parkinsonism: cellular mechanisms of graft-induced functional recovery. Curr Opin Neurobiol 2:683-689.

Björklund A, Stenevi U, Dunnett S, Iversen S (1981) Functional reactivation of the deafferented neostriatum by nigral transplants. Nature 289:497-499.

Björklund A, Stenevi U, Schmidt RH, Dunnett SB, Gage FH (1983a) Intracerebral grafting of neuronal cell suspensions. I. Introduction and general methods of preparation. Acta Physiol Scand 522:1-7.

Björklund A, Stenevi U, Schmidt RH, Dunnett SB. Gage FH (1983b) Intracerebral grafting of neuronal cell suspensions. II. Survival and growth of nigral cell suspensions implanted in different brain sites. Acta Physiol Scand 522:9-18.

Björklund A, Lindvall O, Isacson O, Brundin P, Wictorin K, Strecker RE, Clarke DJ, Dunnett SB (1987) Mechanisms of action of intracerebral neural implants. Trends Neurosci 10:509-516.

Björklund A, Dunnett SB, Nikkhah G (1994) Nigral transplants in the rat Parkinson model: functional limitations and strategies to enhance nigrostriatal reconstruction. In: Functional neural transplantation (Dunnett SB, Björklund A, eds), pp 47-69. New York: Raven.

Bolam JP, Freund TF, Björklund A, Dunnett SB, Smith AD (1987) Synaptic input and local output of dopaminergic neurons in grafts that functionally reinnervate the host neostriatum. Exp Brain Res 68: 131-146.

Bruno JP, Snyder AM, Stricker EM (1984) Effect of dopamine-depleting brain lesions on suckling and weaning in rats. Behav Neurosci 98:156-161.

Bruno JP, Zigmond MJ, Stricker EM (1986) Rats given dopaminedepleting brain lesions as neonates do not respond to acute homeostatic imbalances as adults. Behav Neurosci 100:125-128.

Bruno JP, Jackson D, Zigmond MJ, Stricker EM (1987) Effect of dopamine-depleting brain lesions in rat pups: role of striatal serotonergic neurons in behavior. Behav Neurosci 101:806-811

Carder RK, Snyder KA, Lund RD (1987) Amphetamine- and stressinduced turning after nigral transplants in neonatally dopamine-depleted rats. Dev Brain Res 33:315-318.

Carder RK, Snyder KA, Lund RD (1988) Behavioral and anatomical correlates of immunologically induced rejection of nigral xenografts. J Comp Neurol 277:391-402.

Cenci MA, Björklund A (1994) Transection of corticostriatal afferents abolishes the hyperexpression of Fos induced by intrastriatal dopamine-rich grafts when challenged with amphetamine. Brain Res, in press.

Cenci MA, Kalen P, Duan W-M, Björklund A (1994) Transmitter release from transplants of fetal ventral mesencephalon or locus coeruleus in the rat frontal cortex and nucleus accumbens: effects of pharmacological and behaviorally stimuli. Brain Res 641:225-248.

Cheramy A, Leviel V, Glowinski J (1981) Dendritic release of dopamine in the substantia nigra. Nature 289:537-542.

Choulli K, Herman JP, Rivet JM, Simon H, LeMoal M (1987) Spontaneous and graft-induced behavioral recovery after 6-hydroxydopamine lesion of the nucleus accumbens in the rat. Brain Res 407:376380.

Cunningham MG, McKay RDG (1993) A hypothermic miniaturized stereotaxic instrument for surgery in newborn rats. J Neurosci Methods $47: 105-114$.

Cunningham MG, Nikkhah G, McKay RDG (1993) Grafting immortalized hippocampal cells into the brain of the adult and the newborn rat. Neuroprotocols 3:260-272.

Doncet G. Murata Y, Brundin P, Bosler O, Mons N, Geffard M, Ouimet CC, Björklund A (1989) Host afferents into intrastriatal transplants of fetal ventral mesencephalon. Exp Neurol 106:1-19.

Dunnctt SB, Robbins TW (1992) The functional role of mesotelence phalic dopamine systems. Biol Rev 67:491-518.

Dunnett SB, Björklund A, Schmidt RH, Stenevi U, Iversen SD (1983a) Intracerebral grafting of neuronal cell suspensions. IV. Behavioural recovery in rats with unilateral 6-OHDA lesions following implantation of nigral cell suspensions in different forebrain sites. Acta Physiol Scand 522:29-37.

Dunnett SB, Björklund A, Schmidt RH, Stenevi U, Iversen SD (1983b) Intracerebral grafting of neuronal cell suspensions. V. Behavioural recovery in rats with bilateral 6-OHDA lesions following implantation of nigral cell suspensions. Acta Physiol Scand 522:39-47. 
Feeser HR, Raskin LA (1987) Effects of neonatal dopamine depletion on spatial ability during ontogeny. Behav Neurosci 101:812-818.

Fisher LJ, Young SJ, Tepper JM, Groves PM, Gage FH (1991) Electrophysiological characteristics of cells within mesencephalon suspension grafts. Neuroscience 40:109-122.

Freed CR, Breeze RE, Rosenberg NL, Schneck SA, Kriek E, Qi J-X, Lone TM, Zhang Y-B, Snyder JA, Wells TH, Ramig LO, Thompson L, Mazziotta JC, Huang SC, Grafton ST, Brooks D, Sawle G, Schroter G, Ansari AA (1992) Survival of implanted fetal dopamine cells and neurological improvement 12 to 46 months after transplantation for Parkinson's disease. N Engl J Med 327:1549-1555.

Freund TF, Bolam JP, Björklund A, Stenevi, U, Dunnett, SB, Powell JF Smith AD (1985) Efferent synaptic connections of grafted dopaminergic neurons reinnervating the host neostriatum: a tyrosine hydroxylase immunocytochemical study. J Neurosci 5:603-616.

Gerfen CR (1992) The neostriatal mosaic: multiple levels of compartmental organization. Trends Neurosci 15:133-139.

Herman JP, Choulli K, Geffard M, Nadaud D, Taghzouti K, LeMoal M (1986) Reinnervation of the nucleus accumbens and frontal cortex of the rat by dopaminergic grafts and effects on hoarding behavior. Brain Res 372:210-216.

Herman JP, Rivet JM, Abrous N, LeMoal M (1988) Intracerebral dopaminergic transplants are not activated by electrical footshock stress activating in situ mesocorticolimbic neurons. Neurosci Lett 90:1-2.

Herman JP, Choulli K, Abrous N, LeMoal M (1989) Intracerebral grafts of dopaminergic neurons: a discussion of their functional effects and mechanisms of action. In: Neuronal grafting and Alzheimer's disease (Gage F, Privat A, Christen Y, eds), pp 21-33. Berlin: Springer.

Herman JP, Abrous DN, LeMoal M (1991) Anatomical and behavioral comparison of unilateral dopamine-rich grafts implanted into the striatum of neonatal and adult rats. Neuroscience 40:465-475.

Iwashita Y, Kawaguchi S, Murata M (1994) Restoration of function by replacement of spinal cord segments in the rat. Nature 367:167-170.

Lindvall O, Brundin P, Widner H, Rehncrona S, Gustavii B, Frackowiak R, Leenders KL, Sawle G, Rothwell JC, Marsden CD, Björklund A (1990) Grafts of fetal dopamine neurons survive and improve motor function in Parkinson's disease. Science 247:574-577.

Lund RD, Radel JD, Hankin MH, Klassen H, Coffey PJ, Rawlins JNP (1990) Development and functional integration of retinal transplants with host rat brains. In: Brain repair (Björklund A, Aguayo AJ, Ottoson D, eds), pp 327-340. New York: Stockton.

Lund RD, Radel ID, Coffey PI (1991) The impact of intracerebral retinal transplants on types of behavior exhibited by host rats. Trends Neurosci 14:358-362.

Luthman J, Cowburn R, Grazia De Simoni M, Renyi L (1994) Plasticity responses after neonatal dopamine lesions induced with 6-hydroxydopamine. In: Wenner-Gren international series, Vol 62, Trophic regulation of the basal ganglia. Focus on dopamine neurons (Fuxe K, Agnati LF, Bjelke B, Ottoson D, eds), pp 479-501. Oxford: Pergamon.

Mahalik TJ, Finger TE, Strömberg I, Olson L (1985) Substantia nigra transplants into denervated striatum of the rat: ultrastructure of graft and host interconnections. J Comp Neurol 240:60-70.

Mandel RJ, Brundin P, Björklund A (1990) The importance of graft placement and task complexity for transplant-induced recovery of simple and complex sensorimotor deficits in dopamine denervated rats. Eur J Neurosci 2:888-894.

Mendez I, Elisevich K, Flumerfelt BA (1991) Dopaminergic innervation of substance P-containing striatal neurons by fetal nigral grafts: an ultrastructural double-labeling immunocytochemical study. J Comp Neurol 308:66-78.
Montoya CP, Astell S, Dunnett SB (1990) Effects of nigral and striatal grafts on skilled forelimb use in the rat. Prog Brain Res 82:459-466.

Montoya CP, Campbell HL, Pemberton KD, Dunnett SB (1991) The 'staircase test': a mcasure of independent forclimb reaching and grasping abilities in rats. J Neurosci Methods 36:2-3.

Nikkhah G, Duan W-M, Knappe U, Jödicke A, Björklund A (1993) Restoration of complex semsorimotor behavior and skilled forelimb use by a modified nigral cell suspension transplantation approach in the rat Parkinson model. Neuroscience 56:33-43.

Nikkhah G, Cunningham MG, Jodicke A, Knappe U, Björklund A (1994a) Improved graft survival and striatal reinnervation by microtransplantation of fetal nigral cell suspensions in the rat Parkinson model. Brain Res 633:133-143.

Nikkhah G, Bentlage C, Cunningham MG, Björklund A (1994b) Intranigral fetal dopamine grafts induce behavioral compensation in the rat Parkinson model. J Neurosci 14:3449-3461.

Nikkhah G, Olsson M, Eberhard J, Bentlage C, Cunningham MG, Björklund A (1994c) A microtransplantation approach for cell suspension grafting in the rat Parkinson model. A detailed account of the methodology. Neuroscience 63:57-72.

Nikkhah G, Cunningham MG, Cenci MA, McKay R, Björklund A (1995) Dopaminergic microtransplants into the substantia nigra of neonatal rats with bilateral 6-OHDA lesions. I. Evidence for anatomical reconstruction of the nigrostriatal pathway. J Neurosei 15:35483561.

Robertson GS, Damsma G, Fibiger HC (1991) Characterization of dopamine release in the substantia nigra by in vivo microdialysis in freely moving rats. J Neurosci 11:2209-2216.

Robertson HA (1992) Dopamine receptor interactions: some implications for the treatment of Parkinson's disease. Trends Neurosci 15: 201-206.

Rogers DC, Dunnett SB (1989) Neonatal dopamine-rich grafts and $6-U H D A$ lesions independently provide partial protection from the adult nigrostriatal lesion syndrome. Behav Brain Res 34:1-2.

Rogers DC, Dunnett SB (1990) Nigral grafts in neonatal rats: protection from aphagia induced by subsequent adult 6-OHDA lesions. Prog Brain Res 82:489-492.

Rogers DC, Martel FL, Dunnett SB (1990) Nigral grafts in neonatal rats protect from aphagia induced by subsequent adult 6-OHDA lesions: the importance of striatal location. Exp Brain Res 80:172-176.

Schallert T, Hall S (1988) 'Disengage' sensorimotor deficit following apparent recovery from unilateral dopamine depletion. Behav Brain Res 30:15-24.

Schwarz SS, Freed WJ (1987) Brain tissue transplantation in neonatal rats prevents a lesion-induced syndrome of adipsia, aphagia and akinesia. Exp Brain Res 65:449-454.

Snyder-Keller AM, Lund RD (1990) Amphetamine sensitization of stress-induced turning in animals given unilateral dopamine transplants in infancy. Brain Res 514:143-146.

Snyder-Keller AM, Carder RK, Lund RD (1989) Developnentl of dopamine innervation and turning behavior in dopamine-depleted infant rats receiving unilateral nigral transplants. Neuroscience 30:779-794.

Ungerstedt U, Arbuthnott G (1970) Quantitative recording of rotational behavior in rats after 6-hydroxy-dopamine lesions of the nigrostriatal dopamine system. Brain Res 24:485-493.

Whishaw IQ, Funk DR, Hawryluk SJ, Karbashewski ED (1987) Absence of sparing of spatial navigation, skilled forelimb and tongue use and limb posture in the rat after neonatal dopamine depletion. Physiol Behav 40:247-253.

Widner H, Tetrud J, Rehncrona S, Snow B, Brundin P, Gustavii B, Björklund A, Lindvall O, Langston J (1992) Bilateral fetal mesencephalic grafting in two patients with parkinsonism induced by 1-methyl-4-phenyl-1,2,3,6-tetrahydropyridine (MPTP). N Engl J Med 327:1556-1563. 